A Population Explosion: The Nature and Evolution of X-ray Binaries in Diverse Environments

28 Oct.-2 Nov. 2007, St. Petersburg Beach, FL

R.M. Bandyopadhyay, S. Wachter, D. Gelino, \& C. R. Gelino, eds.

Session: Population Synthesis

\title{
X-Ray Binaries and the Dynamical States of Globular Clusters
}

\author{
John M. Fregeau \\ Chandra Fellow; Department of Physics and Astronomy, Northwestern University, \\ Evanston, IL 60208
}

\begin{abstract}
We summarize and discuss recent work (Fregeau 2007) that presents the confluence of three results suggesting that most Galactic globular clusters are still in the process of core contraction, and have not yet reached the thermal equilibrium phase driven by binary scattering interactions: that 1) the three clusters that appear to be overabundant in X-ray binaries per unit encounter frequency are observationally classified as "core-collapsed," 2) recent numerical simulations of cluster evolution with primordial binaries show that structural parameters of clusters in the binary-burning phase agree only with "core-collapsed" clusters, and 3) a cluster in the binaryburning phase for the last few Gyr should have $\sim 5$ times more dynamically formed X-ray sources than if it were in the core contraction phase for the same time.
\end{abstract}

\section{Introduction}

This proceedings article briefly summarizes my contributed talk at the "Population Explosion" meeting, which itself was a brief summary of the work described in Fregeau (2007). I have taken the opportunity here to include supplemental material, but first refer the reader to the detailed, complete discussion in Fregeau (2007).

\subsection{X-Ray Sources and Cluster Dynamics}

Although X-ray binaries have been known to be overabundant in Galactic globular clusters (by unit mass relative to the disk) for over 30 years (Clark 1975; Katz 1975), it is only recently that clear evidence for their dynamical origin has revealed itself. Pooley et al. (2003) found that the number of X-ray sources with $L_{X}>4 \times 10^{30} \mathrm{erg} / \mathrm{s}, N_{X}$, in a cluster follows a clear, nearly linear correlation with the encounter frequency $\Gamma$, a measure of the dynamical interaction rate in the cluster. There are a few clear outliers to this trend, however. Terzan 1 is overabundant in $N_{X}$ by a factor of $\sim 20$, NGC 6397 by a factor of $\sim 5$, and NGC 7099 by a factor of $\sim 2$ (Cackett et al. 2006; Lugger et al. 2007). The common thread among these clusters is that they are all classified observationally as "core-collapsed," while the other clusters in the sample are not. A cluster is observationally termed core-collapsed if its surface brightness profile is consistent with a cusp at the limit of observational resolution. Since this observational classification is linked with the cluster evolutionary state (as described below), it appears that cluster evolution complicates the $N_{X}-\Gamma$ correlation.

\subsection{Understanding Cluster Core Radii}

The evolution of a globular cluster, being a bound self-gravitating system, is very similar to that of a star, and comprises three main phases. The cluster first "core contracts" on a relaxation timescale. Once the core density becomes large enough for binary scattering interactions to begin generating energy, the cluster settles into the "binary-burning" phase, 
in which the cluster's core properties remain roughly constant with time. Once the binary population is exhausted in the core, it collapses via the gravothermal instability, leading to extremely high central densities, followed by a series of gravothermal oscillations in which the core expands and contracts repeatedly. (For a graphical representation of the three main phases of cluster evolution, see Figure 1 of Gao et al. (1991), Figure 5 of Fregeau et al. (2003), or Figure 29.1 of Heggie \& Hut (2003).) Recent comparison of star cluster evolution simulations with observations of cluster structural parameters have shown that the clusters observationally classified as core-collapsed are in fact most likely in the binary-burning phase, while the rest are most likely still in the core-contraction phase (Heggie et al. 2006; Fregeau \& Rasio 2007).

\subsection{A Refined $\Gamma$}

First introduced by Verbunt \& Hut (1987), the encounter frequency $\Gamma$ is an estimate of the current dynamical interaction rate in the cluster, which is assumed to be proportional to the current number of observable X-ray sources. Typically, it is approximated as

$$
\Gamma \equiv \frac{d N_{\text {int }}}{d t} \propto \rho_{c}^{2} r_{c}^{3} / v_{\sigma}
$$

where $r_{c}$ is the cluster core radius, $\rho_{c}$ is the core mass density, and $v_{\sigma}$ is the core velocity dispersion. More accurate approximations of the interaction rate have been used, including numerical integrals over cluster models (Pooley et al. 2003), but all are estimates of the current interaction rate. Recent work has shown that dynamically formed X-ray binaries have finite detectable lifetimes ( $\sim 10^{5}$ to $\sim 10^{9} \mathrm{yr}$, depending on binary type), and furthermore that there is a lag time of several Gyr between dynamical interaction and the binary turning on as an X-ray source (Ivanova et al. 2006, 2007). Since the binary interaction rate is a function of the cluster properties (density, velocity dispersion, etc.), it is clear that the Xray binaries we see in clusters today were formed several Gyr ago, and thus encode the recent cluster evolution history in their populations. We thus adopt a refined version of $\Gamma$ which encodes this history by integrating the interaction rate over the dynamical evolution of the cluster. The resulting quantity is the total number of strong dynamical interactions, which should be roughly proportional to the currently observable number of X-ray binaries. Plugging in the different phases of cluster evolution will yield different estimates for the number of sources, and possibly allow one to differentiate among the different phases by comparing with the observed number of sources. Using standard assumptions, and adopting some results from recent $N$-body simulations for the evolution of $r_{c}$ with time, one finds the ratio of the number of interactions for a cluster in the binary-burning phase to one in the core contraction phase is

$$
\frac{N_{\mathrm{int}, \mathrm{bb}}}{N_{\mathrm{int}, \mathrm{cc}}}=\frac{t_{x}}{t_{0}} \frac{2.835}{\left(\frac{t_{0}}{t_{0}+9 t_{\ell}}\right)^{0.315}-\left(\frac{t_{0}}{t_{0}+9 t_{\ell}+9 t_{x}}\right)^{0.315}},
$$

where $t_{x}$ is the $\mathrm{X}$-ray source lifetime, $t_{\ell}$ is the lag time between strong dynamical interaction and X-ray turn-on, and $t_{0}$ is the current cluster age. (The gravothermal oscillation phase is excluded from the discussion since in this phase the core binary fraction would be much smaller than what is observed.) This expression has a minimum of 2.0 and a maximum of 17.8 in the range $t_{x}=10^{-4}-3 \mathrm{Gyr}, t_{\ell}=1-10 \mathrm{Gyr}$, for $t_{0}=13 \mathrm{Gyr}$. For the canonical values of $t_{x}=1 \mathrm{Gyr}$ and $t_{\ell}=3 \mathrm{Gyr}$ with $t_{0}=13 \mathrm{Gyr}$, the value is 5.0 . Since the number of X-ray sources should scale roughly linearly with the number of interactions, this suggests that if a cluster is in the binary-burning phase (and has been for a time $t_{\ell}+t_{x}$ to the 
present), it should have $\sim 5$ times as many X-ray sources than it would if it were in the core contraction phase. The three observationally core-collapsed clusters in the sample previously mentioned are overabundant in $N_{X}$ by factors compatible with the range of values allowed by this expression, suggesting that the observationally core-collapsed clusters are indeed in the binary-burning phase, while the rest are still in the process of core contraction.

\subsection{Discussion}

Since only $\sim 20 \%$ of Galactic globular clusters are observationally classified as core-collapsed, the conclusion that seems strongly suggested is that most clusters $(\sim 80 \%)$ are currently still in the core contraction phase, while those that are core-collapsed are in the binary burning phase. This goes counter to the widely held belief that most clusters are currently in the binary burning phase, and complicates the many existing studies that have assumed cluster core properties that are constant with time, including predictions of blue straggler populations, tidal-capture binaries, and the evolution of the core binary fraction (e.g., Mapelli et al. 2004; Di Stefano \& Rappaport 1994; Ivanova et al. 2005). Similarly, this result does away with the need for alternate energy sources in cluster cores to explain currently observed core radii, including intermediate-mass black holes in most clusters, mass loss from stellar mergers, and ongoing mass segregation (Trenti 2006; Chatterjee et al. 2007; Merritt et al. 2004).

\section{Meta-Discussion}

A few interesting aspects of this analysis did not fit into the original letter. It has been pointed out that an important but neglected factor going into the interaction rate is the neutron star retention fraction, since it depends on the cluster escape speed, and since it is neutron star + stellar binary interactions that predominantly lead to X-ray binaries (Rasio, private communication). Since there is no reason to expect that the cluster escape speed at the time of neutron star formation does not vary from cluster to cluster, the correlation between dynamical interaction rate and $N_{X}$ should be blurred significantly by this effect. We note that the analysis in Fregeau (2007) avoids this issue entirely by comparing a cluster only with a version of itself in a different evolutionary phase.

Another issue is that there is apparently a significant contribution to the X-ray binary population from primordial binaries in clusters with small encounter frequencies. (This is likely the reason for the sub-linear dependence of $N_{X}$ on $\Gamma$ in Pooley et al. (2003).) This has given rise to the use of the interaction rate per unit cluster mass, $\gamma \equiv \Gamma / M_{\text {clus }}$, to help isolate the dynamically-formed population in the analysis. The use of $\gamma$ has, for example, shown that a significant fraction of cataclysmic variables are dynamically formed (Pooley \& Hut 2006). The results of Fregeau (2007) could be further tested in the future with the more sensitive $\gamma$ as the library of deep X-ray observations of globular clusters grows.

Finally, we mention that in the near future we plan to test our simple overabundance prediction by using the detailed numerical models of Ivanova et al. (2005). Although there are some hints from that paper that inputting a time-varying core does not noticeably affect the final core binary fraction, we expect that the number of visible X-ray binaries, while noisy, may be more noticeably affected.

Acknowledgments. JMF acknowledges support from Chandra theory grant TM6-7007X and Chandra Postdoctoral Fellowship Award PF7-80047. 
Fregeau; X-Ray Binaries and the Dynamical States of Globular Clusters

\section{References}

Cackett, E. M., Wijnands, R., Heinke, C. O., Pooley, D., Lewin, W. H. G., Grindlay, J. E., Edmonds, P. D., Jonker, P. G., \& Miller, J. M. 2006, MNRAS, 369, 407

Chatterjee, S., Fregeau, J. M., \& Rasio, F. A. 2007, IAU Symposium 246, 2007, Capri, Italy, Poster \#?

Clark, G. W. 1975, ApJ, 199, L143

Di Stefano, R. \& Rappaport, S. 1994, ApJ, 423, 274

Fregeau, J. 2007, ApJL (in press), arXiv:0710.4556

Fregeau, J. M., Gürkan, M. A., Joshi, K. J., \& Rasio, F. A. 2003, ApJ, 593, 772

Fregeau, J. M. \& Rasio, F. A. 2007, ApJ, 658, 1047

Gao, B., Goodman, J., Cohn, H., \& Murphy, B. 1991, ApJ, 370, 567

Heggie, D. \& Hut, P. 2003, The Gravitational Million-Body Problem (Cambridge University Press, 2003, 372 p.)

Heggie, D. C., Trenti, M., \& Hut, P. 2006, MNRAS, 368, 677

Ivanova, N., Belczynski, K., Fregeau, J. M., \& Rasio, F. A. 2005, MNRAS, 358, 572

Ivanova, N., Heinke, C., Rasio, F. A., Belczynski, K., \& Fregeau, J. 2007, ArXiv e-prints, 706

Ivanova, N., Heinke, C. O., Rasio, F. A., Taam, R. E., Belczynski, K., \& Fregeau, J. 2006, MNRAS, 372,1043

Katz, J. I. 1975, Nat, 253, 698

Lugger, P. M., Cohn, H. N., Heinke, C. O., Grindlay, J. E., \& Edmonds, P. D. 2007, ApJ, 657, 286

Mapelli, M., Sigurdsson, S., Colpi, M., Ferraro, F. R., Possenti, A., Rood, R. T., Sills, A., \& Beccari, G. 2004, ApJ, 605, L29

Merritt, D., Piatek, S., Portegies Zwart, S., \& Hemsendorf, M. 2004, ApJ, 608, L25

Pooley, D. \& Hut, P. 2006, ApJ, 646, L143

Pooley, D., Lewin, W. H. G., Anderson, S. F., Baumgardt, H., Filippenko, A. V., Gaensler, B. M., Homer, L., Hut, P., Kaspi, V. M., Makino, J., Margon, B., McMillan, S., Portegies Zwart, S., van der Klis, M., \& Verbunt, F. 2003, ApJ, 591, L131

Trenti, M. 2006, ArXiv Astrophysics e-prints

Verbunt, F. \& Hut, P. 1987, in IAU Symposium, Vol. 125, The Origin and Evolution of Neutron Stars, ed. D. J. Helfand \& J.-H. Huang, 187 